\title{
Basal cell carcinoma in the inner canthus
}

\author{
Boris Jančar \\ Department of Radiation Oncology, Institute of Oncology, Ljubljana, Slovenia
}

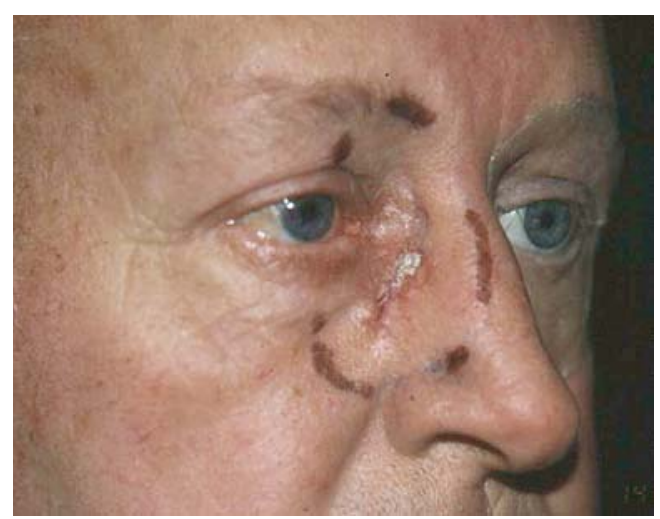

Figure 1. Basal cell carcinoma in the inner canthus of the right orbit; irradiation field is marked.

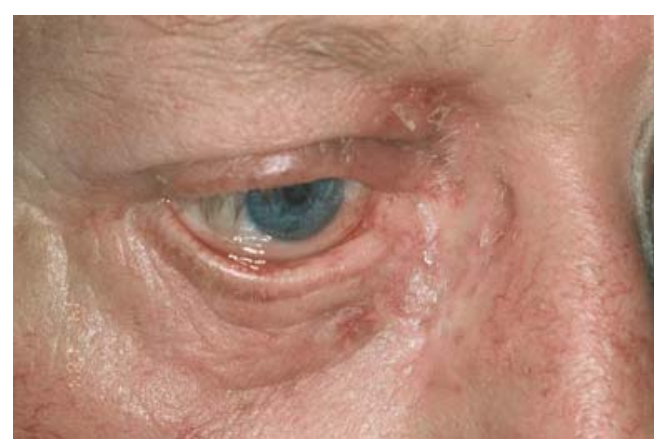

Figure 3. One year after irradiation.

Received 12 April 2007

Accepted 19 April 2007

Correspondence to: Prim. Boris Jančar, MD, MSc, Department of Radiation Oncology, Institute of Oncology Ljubljana, Zaloška 2, Ljubljana, Slovenia; Phone; + 38615879 295; Fax: + 38615879 295; E-mail: bojancar@onko-i.si

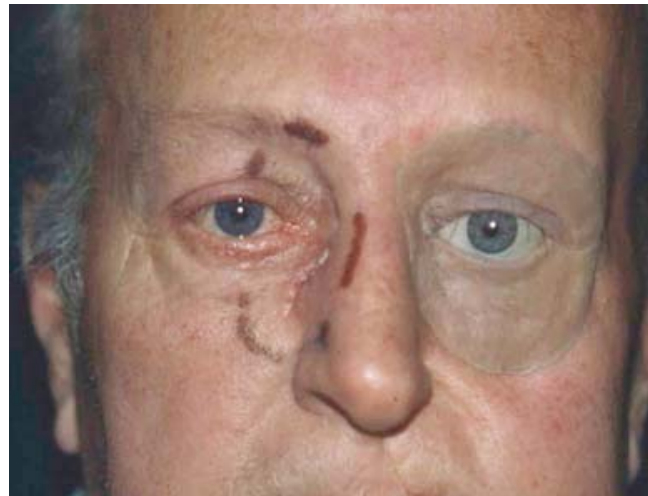

Figure 2. Tumour in the inner canthus of the right eye and epithesis of the left orbit and ethmoid.

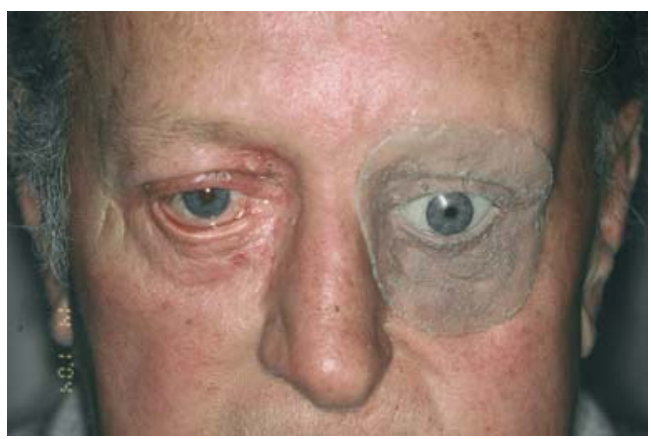

Figure 4. One year after irradiation.

In January 2003, a 66-year-old patient visited the outpatient clinic at our Institute for the follow-up examination of the tumour in the inner canthus of the right eye. Biopsy of the tumour was made in November 2002 at the Department of Otorhinolaryngology and Cervicofacial Surgery of the University Medical Centre Ljubljana. Histology of the 
excised sample confirmed basal cell carcinoma. The patient also told that, 10 years earlier, i.e. in 1993, he was operated on for a "lump" that developed on the same site. The patient was told that "lump" was sebaceous glad. But in a year, the "lump" reappeared and was removed again.

In 1994, the patient was operated on for squamous cell carcinoma of the ethmoidal sinus on the right, which was infiltrating into the right orbit. Ethmoidectomy was performed on the right side, with the exenteration of the right orbit. Postoperatively, the patient was treated also with telecobalt therapy and received a tumour dose (TD) 56 Gy.
On the follow-up examination in 2003, an indurated $2.5 \mathrm{~cm}$ long scar was still seen in the canthus of the right eye. The left cheek and orbit were covered with epithesis material (Figures 1, 2). The patient was irradiated by an orthovolt unit (Pantak), with the bulb of the irradiated eye protected; he received a TD 40 Gy. The tumour regressed completely (Figures 3,4). These photos (Figures $3,4)$ were taken on 14 January 2004.

On the last follow-up control in May 2006, no evidence of the disease recurrence was observed either in the area of ethmoid sinus or in the inner canthus of the right eye. 